\title{
Time-Orthogonal Unitary Dilations and Noncommutative Feynman-Kac Formulae
}

\author{
R. L. Hudson ${ }^{\star 1}$, P. D. F. Ion ${ }^{2}$, and K. R. Parthasarathy ${ }^{{ }^{3}}$ \\ 1 Mathematics Department, University of Nottingham, University Park, Nottingham NG7 2RD, \\ England \\ 2 Mathematical Reviews, University of Michigan, 611 Church Street, Ann Arbor, MI 48109, USA \\ 3 Indian Statistical Institute, 7, S.J.S. Sansanwal Marg, New Dehli-110016, India
}

\begin{abstract}
An analysis of Feynman-Kac formulae reveals that, typically, the unperturbed semigroup is expressed as the expectation of a random unitary evolution and the perturbed semigroup is the expectation of a perturbation of this evolution in which the latter perturbation is effected by a cocycle with certain covariance properties with respect to the group of translations and reflections of the line. We consider generalisations of the classical commutative formalism in which the probabilistic properties are described in terms of noncommutative probability theory based on von Neumann algebras. Examples of this type are generated, by means of second quantisation, from a unitary dilation of a given self-adjoint contraction semigroup, called the time orthogonal unitary dilation, whose key feature is that the dilation operators corresponding to disjoint time intervals act nontrivially only in mutually orthogonal supplementary Hilbert spaces.
\end{abstract}

\section{Introduction}

For any positive-self-adjoint operator $H$ in a Hilbert space $h_{0}$ we construct a timeorthogonal unitary dilation $\left\{U_{\mathrm{s}, t}, s \geqq t\right\}$ of the contraction semigroup $\left\{e^{-t H}, t \geqq 0\right\}$ in the Hilbert space $h_{0} \oplus L_{2}\left(\mathbb{R}_{0} h_{0}\right)$ which exhibits the following properties:

(1) the family $\left\{U_{s, t} s \geqq t\right\}$ is a strongly continuous unitary evolution in the sense that $U_{r, s} U_{s, t}=U_{r, t}$ for all $r \geqq s \geqq t$;

(2) it is time-orthogonal in the sense that $U_{s, t}$ acts nontrivially only in the subspace $h_{0} \oplus h_{[s, t]}$ where $h_{]_{\mathrm{s}, t]}} \subset L_{2}\left(\mathbb{R}, h_{0}\right)$ is the subspace of functions with support contained in $] s, t]$;

(3) it is covariant under time translations and time reversal. This dilation is somewhat different from the classical dilations [14] of a one parameter con-

* Parts of this work were carried out when the first author was a participant in the Sonderforschungsbereich 123 (Stochastische Mathematische Modelle) of the DeutscheForschungsgemeinschaft and when the third author was Visiting Fellow at the University of Warwick 
traction semigroup into a one parameter group, examples of which may be found in $[10,4,5]$.

Using the unitary evolution induced by second quantisation of the above dilation in the Fock space $\Gamma\left(h_{0} \oplus L_{2}\left(\mathbb{R}, h_{0}\right)\right)$ and contracting a suitable perturbation of this new evolution to the smaller Fock space $\Gamma\left(h_{0}\right)$ we obtain a noncommutative version of the Feynman-Kac formula for the semigroup $\exp \{-t(d \Gamma(H)+V)\}$ where $V$ is a bounded operator in $\Gamma\left(\hat{\hbar}_{0}\right)$ and $\exp \{-t d \Gamma(H)\}$ $=\Gamma(\exp (-t H)), \Gamma$ being the second quantisation map. For an alternative version of a Feynman-Kac formula based on classical dilations and completely positive maps in the theory of quantum stochastic processes the reader may refer to Accardi [1].

We use the following conventions. A product $\prod_{j=1}^{n} A_{j}$ of noncommuting elements is taken in the order $A_{n} A_{n-1} \ldots A_{1}$. A canonical pair is a pair $(p, q)$ of selfadjoint operators satisfying the Weyl relation

$$
e^{i x p} e^{i y q}=e^{i x y} e^{i y q} e^{i x p}, \quad x, y \in \mathbb{R} .
$$

We shall say that von Neumann algebras $N_{1}, N_{2}, \ldots, N_{n}$ in a Hilbert space $h$ are independent (in the state $\Omega$ ) if $\Omega$ is a unit vector in $h$ for which

$$
\left\langle\Omega, \prod_{j=1}^{n} A_{j} \Omega\right\rangle=\prod_{j=1}^{n}\left\langle\Omega, A_{j} \Omega\right\rangle, \quad A_{j} \in N_{j}, \quad j=1, \ldots, n .
$$

If $h_{0}$ is a second Hilbert space then the conditional expectation of $A \in \mathbf{B}\left(h_{0} \otimes h\right)$ given $\mathbf{B}\left(h_{0}\right)$ is the element $\mathbb{E}\left[A \mid \mathbf{B}\left(h_{0}\right)\right] \in \mathbf{B}\left(h_{0}\right)$ for which

$$
\left\langle\psi, \mathbb{E}\left[A \mid \mathbf{B}\left(h_{0}\right)\right] \chi\right\rangle=\langle\psi \otimes \Omega, A \chi \otimes \Omega\rangle .
$$

The following theorem is almost obvious [8].

Theorem 1.1. Let $N_{1}, N_{2}, \ldots, N_{n}$ be independent von Neumann algebras and set

$$
N^{(j)}=\mathbf{B}\left(\hbar_{0}\right) \otimes N_{j}, \quad j=1, \ldots, n .
$$

Then, for arbitrary $A^{(j)} \in N^{(j)}, j=1, \ldots, n$,

$$
\mathbb{E}\left[\prod_{j=1}^{n} A^{(j)} \mid \mathbf{B}\left(\ell_{0}\right)\right]=\prod_{j=1}^{n} \mathbb{E}\left[A^{(j)} \mid \mathbf{B}\left(\ell_{0}\right)\right] .
$$

\section{Evolutions and Perturbations}

Let $h$ be a separable Hilbert space and let $\left(U_{s, t}: s \geqq t\right)$ be a family of bounded operators on $h$ satisfying the conditions

a) $U_{s, t}$ is strongly continuous in $s$ for fixed $t$ and in $t$ for fixed $s$.

b) $U_{s, s}=I$ for all $s \in \mathbb{R}$.

c) $U_{r, s} U_{s, t}=U_{r, t}$ for $r \geqq s \geqq t$.

Such a family $\left(U_{s, t}\right)$ is called an evolution. If each $U_{s, t}$ is unitary (respectively self-adjoint, a contraction) then the evolution is said to be unitary (respectively self-adjoint, contractive). If $U_{s, t}=U_{s-t}$ depends only on the difference $s-t$ then the 
evolution is a semigroup. Every (strongly continuous) self-adjoint semigroup is of the form $U_{s-t}=\exp \{-(s-t) H\}$ where $H$ is a not necessarily bounded self-adjoint operator called its infinitesimal generator.

A family $\left(M_{s, t}: s \geqq t\right)$ of bounded operators in $h$, strongly continuous in $s$ and $t$ separately and satisfying $M_{s, s}=I$ for all $s \in \mathbb{R}$ is said to be a cocycle for the evolution $\left(U_{s, t}: s \geqq t\right)$ if, for $r \geqq s \geqq t$,

$$
M_{r, t}=M_{r, s} U_{r, s} M_{s, t} U_{r, s}^{-1} .
$$

It is easy to see that if $\left(M_{s, t}\right)$ is a cocycle for the unitary evolution $\left(U_{s, t}\right)$ and if, for $s \geqq t$,

$$
\tilde{U}_{s, t}=M_{s, t} U_{s, t},
$$

then $\left(\tilde{U}_{s, t}, s \geqq t\right)$ is also an evolution; indeed (2.1) is equivalent to the defining condition c) and continuity follows from the strong continuity of operator multiplication when one of the factors is uniformly bounded. We call the evolution $\left(\tilde{U}_{s, t}\right)$ the perturbation of $\left(U_{s, t}\right)$ by the cocycle $\left(M_{s, t}\right)$.

The following theorem can be proved using the Picard method of successive approximations to approximate $M_{s, t}^{B}$ in operator-bound norm by $M_{s, t}^{(n)}$, defined inductively by $M_{s, t}^{(0)}=I$,

$$
M_{s, t}^{(n)}=I-\int_{t}^{s} M_{s, r}^{(n-1)} U_{s, r} B U_{s, r}^{-1} d r .
$$

See also [12].

Theorem 2.1. Let $\left(U_{s, t}\right)$ be an unitary evolution in the separable Hilbert space ho and let $B$ be a bounded operator in $h$. Then there exists a unique cocycle $\left(M_{s, t}^{B}: s \geqq t\right)$ such that $M_{s, t}^{B}$ is strongly differentiable in $t$ and

$$
\partial_{t} M_{s, t}^{B}=-M_{s, t}^{B} U_{s, t} B U_{s, t}^{-1} .
$$

Moreover if the family $\left(U_{s, t} B U_{s, t}^{-1}: s \geqq t\right)$ is commutative, then the strong operator valued integral $\int_{t}^{s} U_{s, \tau} B U_{s, \tau}^{-1} d \tau$ exists and, for $s \geqq t$,

$$
M_{s, t}^{B}=\exp \left\{-\int_{t}^{s} U_{s, t} B U_{s, \tau}^{-1} d \tau\right\} .
$$

We shall call $\left(M_{s, t}^{B}\right)$ the cocycle determined by $B$ and the corresponding evolution $\left(U_{s, t}^{B}\right)$, with

$$
U_{s, t}^{B}=M_{s, t}^{B} U_{s, t}
$$

the perturbed evolution determined by $B$.

\section{Reduced Evolutions and Semigroups}

Let $h$ be a separable Hilbert space. A doubly filtered von Neumann algebra in $h$ is a pair $\left(N,\left(N_{s, t}: s \geqq t\right)\right)$ comprising a von Neumann algebra $N$ and a system $\left(N_{s, t}: s \geqq t\right)$ 
of von Neumann subalgebras which generate $N$ as a von Neumann algebra and which satisfy the condition

$$
\left.\left.\left.\left.N_{s, t} \subset N_{a, b} \text { whenever }\right] \mathrm{s}, t\right] \leqq\right] a, b\right] .
$$

A covariant system is a triple $\left(N,\left(N_{s, t}: s \geqq t\right),\left(\gamma_{t}: t \in \mathbb{R}\right)\right)$ consisting of a doubly filtered von Neumann algebra $\left(N,\left(N_{s, t}: s \geqq t\right)\right)$ and a one-parameter group $\left(\gamma_{t}\right)$ of normal automorphisms of $N$ satisfying

$$
\gamma_{r}\left(N_{s, t}\right) \subseteq N_{r+s, r+t} \text {. }
$$

A reflective covariant system is a quadruple $\left(N,\left(N_{s, t}\right),\left(\gamma_{t}\right), \varrho\right)$ consisting of a covariant system $\left(N,\left(N_{s, t}\right),(\gamma)\right)$ together with a normal automorphism $\varrho$ of $N$ satisfying, for $r \in \mathbb{R}$ and $s \geqq t$,

$$
\begin{gathered}
\varrho \gamma_{r}=\gamma_{-r} \varrho, \quad \varrho=\varrho^{-1} \\
\varrho N_{s, t} \cong N_{-t,-s} .
\end{gathered}
$$

Let $h_{0}$ be a second Hilbert space. A reducing map for a doubly filtered von Neumann algebra $\left(N,\left(N_{s, t}\right)\right)$ is a strongly continuous linear map $j$ from $N$ to $B\left(h_{0}\right)$ such that, for all $A \in N$,

$$
j(A)^{*}=j\left(A^{*}\right)
$$

and for $A \in N_{a, b}, B \in N_{c, d}$ with $\left.\left.\left.] a, b\right] \cap\right] c, d\right]=\emptyset$,

$$
j(A B)=j(A) j(B) .
$$

A covariant reducing map for a covariant system $\left(N,\left(N_{s, t}\right),\left(\gamma_{t}\right)\right)$ is a reducing map $j$ for $\left(N,\left(N_{s, t}\right)\right)$ for which $j \circ \gamma_{r}=j$ for all $r \in \mathbb{R}$, and a reflective covariant reducing map for a reflective covariant system $\left(N,\left(N_{s, t}\right),\left(\gamma_{t}\right), \varrho\right)$ is a covariant reducing map for $\left(N,\left(N_{s, t}\right),\left(\gamma_{t}\right)\right)$ for which $j \circ \varrho=\varrho$.

An evolution $\left(U_{s, t}\right)$ in $h$ is said to be adapted to the doubly filtered von Neumann algebra $\left(N,\left(N_{s, t}\right)\right)$ if

$$
U_{s, t} \in N_{s, t}
$$

for all $s \geqq t$, covariantly adapted for the covariant system $\left(N,\left(N_{s, t}\right),\left(\gamma_{t}\right)\right)$ if, in addition to (3.2), for all $r \in \mathbb{R}$ and $s \geqq t$

$$
\gamma_{r}\left(U_{s, t}\right)=U_{r+s, r+t},
$$

and reflectively covariantly adapted for the reflective covariant system $\left(N,\left(N_{s, t}\right),\left(\gamma_{t}\right), \varrho\right)$ if, in addition to (3.2) and (3.3),

$$
\varrho\left(U_{s, t}^{*}\right)=U_{-t,-s} .
$$

It is clear from (3.1) that if $\left(U_{s, t}\right)$ is an adapted evolution and $j$ is a reducing map for $\left(N,\left(N_{s t}\right)\right)$

$$
U_{\mathrm{s}, t}^{(j)}=j\left(U_{s, t}\right)
$$

defines an evolution $\left(U_{s, t}^{(j)}: s \geqq t\right)$ in $h_{0}$ called the reduced evolution corresponding to $j$. 
Theorem 3.1. (a) The reduced evolution of a covariantly adapted evolution corresponding to a covariant reducing map is a semigroup.

(b) The reduced evolution of a reflectively covariantly adapted evolution corresponding to a reflective covariant reducing map is a self-adjoint semigroup.

Proof. (a) If $\left(U_{s, t}\right)$ is covariantly adapted and $j$ is a covariant reducing map then, for $r \in \mathbb{R}, s \geqq t$

$$
\begin{aligned}
U_{r+s, r+t}^{(j)} & =j\left(U_{r+s, r+t}\right) \\
& =j \circ \gamma_{r}\left(U_{s, t}\right) \\
& =j\left(U_{s, t}\right) \\
& =U_{s, t}^{(j)}
\end{aligned}
$$

showing that $U_{s, t}^{(j)}$ depends only on the difference $s-t$ and hence that the reduced evolution is a semigroup.

(b) If in addition $\left(U_{s, t}\right)$ is reflectively covariantly adapted and $j$ is a reflective covariant reducing map then, for $s \geqq t$,

$$
\begin{aligned}
{\left[U_{s, t}^{(j)}\right]^{*} } & =\left[j\left(U_{s, t}\right)\right]^{*} \\
& =j\left(U_{s, t}^{*}\right) \\
& =j \circ \varrho\left(U_{-t,-s}\right) \\
& =j\left(U_{-t,-s}\right) \\
& =U_{-t,-s}^{(j)} .
\end{aligned}
$$

Thus, using the semigroup property, $U_{s, t}^{(j)}$ is self-adjoint.

Now we examine the reduction of perturbed evolutions.

Theorem 3.2. (a) Let $\left(N,\left(N_{s, t}\right)\right)$ be a doubly filtered von Neumann algebra and let $B$ be an element of $\bigcap_{s \geqq t} N_{s, t}$. Then, if $\left(U_{s, t}\right)$ is an adapted evolution then the perturbed evolution $\left(U_{s, t}^{B}\right)$ is adapted.

(b) If in addition $\left(N,\left(N_{s, t}\right),\left(\gamma_{t}\right)\right)$ is a covariant system, $\left(U_{s, t}\right)$ is covariantly adapted and $B$ is invariant under each $\gamma_{t}$, then the perturbed evolution $\left(U_{s, t}^{B}\right)$ is covariantly adapted.

(c) If in addition $\left(N,\left(N_{s, t}\right),\left(\gamma_{t}\right), \varrho\right)$ is a reflective covariant system, $\left(U_{s, t}\right)$ is reflectively covariantly adapted, and $B$ is self adjoint, then the semigroup reduced evolution obtained from $\left(U_{s, t}^{B}\right)$ from a reflective covariant reducing map $j$ is selfadjoint with infinitesimal generator

$$
H^{B}=H+j(B),
$$

where $H$ is the infinitesimal generator of the reduced unperturbed evolution.

Proof. (a) To prove that $\left(U_{s, t}^{B}\right)$ is adapted we must show that, for $s \geqq t, M_{s, t}^{B} \in N_{s, t}$. Recalling from Theorem 2.1 that $M_{s, t}^{B}$ was the uniform limit of operators $M_{s, t}^{(n)}$ defined by (2.2), it is sufficient to show that each $M_{s, t}^{(n)} \in N_{s, t}$. Since for $t \leqq \tau \leqq S$, $N_{s, t} \leqq N_{s, t}$ and $U_{s, \tau}, B \in N_{s, \tau}$, the required conclusion follows by induction. 

$s \geqq t$

(b) To prove that $\left(U_{s, t}^{B}\right)$ is covariantly adapted we must show that, for $r \in \mathbb{R}$,

$$
\gamma_{r}\left(M_{s, t}^{B}\right)=M_{r+s, r+t}^{B} .
$$
$M_{s, t}^{(n)}$

It is sufficient to prove the corresponding property for the approximations

$$
\gamma_{r}\left(M_{s, t}^{(n)}\right)=M_{r+s, r+t}^{(n)}, \quad n=0,1,2, \ldots, s \geqq t .
$$

The case $n=0$ is clear since each $M_{s, t}^{(0)}=I$; assuming (3.5) for $n=k-1$ we have, using (2.2),

$$
\begin{aligned}
\gamma_{r}\left(M_{s, t}^{(k)}\right) & =I-\gamma_{r}\left\{\int_{t}^{s} M_{s, \tau}^{(k-1)} U_{s, \tau} B U_{s, \tau}^{-1} d \tau\right\} \\
& =I-\int_{t}^{s} \gamma_{r}\left(M_{s, \tau}^{(k-1)}\right) \gamma_{r}\left(U_{s, \tau}\right) B \gamma\left(U_{s, \tau}\right)^{-1} d \tau \\
& =I-\int_{t}^{s} M_{r+s, r+\tau}^{(k-1)} U_{r+s, r+\tau} B U_{r+s, r+\tau}^{-1} d \tau \\
& =I-\int_{r+t}^{r+s} M_{r+s, \tau}^{(k-1)} U_{r+s, \tau} B U_{r+s, \tau}^{-1} d \tau \\
& =M_{r+s, r+t}^{(k)}
\end{aligned}
$$

and the result follows by induction.

(c) By (b) and Theorem 3.1 (a) we know that the reduced evolution $\left(\left(U_{s, t}^{B}\right)^{j}\right)$ is a semigroup so that we can write

$$
\left(U_{s, t}^{B}\right)^{j}=T_{s-t}
$$

If $f$ is in the domain $D(H)$ of $H$ we have, for $t>0$,

$$
\begin{aligned}
t^{-1}\left(T_{t}-I\right) f & =t^{-1} j\left(M_{0,-t}^{(B)} U_{0,-t}-I\right) f \\
& =j\left(t^{-1}\left(M_{0,-t}^{(B)} U_{0,-t}-U_{0,-t}\right) f+t^{-1} j\left(U_{0,-t}-I\right) f .\right.
\end{aligned}
$$

Since $j$ is strongly continuous and $f \in D(H)$ we can pass to the limit as $t \rightarrow 0$, using (2.3), to conclude that

$$
\lim _{t \downarrow 0} t^{-1}\left(T_{t}-I\right) f=-j(B) f-H f .
$$

But this means that ( $T_{t}$ is the one-parameter semigroup of which the self-adjoint operator $j(B)+H$ is the infinitesimal generator; in particular the reduced semigroup is self-adjoint.

We may summarise Theorem 3.2 (c) by the statement

$$
e^{-(s-t)(H+j(B))}=j\left(M_{s, t}^{B} U_{s, t}\right)
$$

whenever $\left(U_{s, t}\right)$ is a reflectively covariantly adapted evolution, $j$ is a reflective covariant reducing map, and

$$
e^{-(s-t) H}=j\left(U_{s, t}\right)
$$




\section{The Classical Case Generalised to Imprimitivity Systems}

Let $G$ be a complete, separable metric group acting on a Borel space $(X, \mathscr{B})$ and let $h_{0}$ be a separable Hilbert space. Suppose that $g \rightarrow U_{g}$ is a strongly continuous unitary representation of $G$ in $h_{0}$ and $A \rightarrow E(A)$ is a spectral measure on $\mathscr{B}$ relative to $h_{0}$ satisfying

$$
U_{g} E(A) U_{g}^{-1}=E(g A) \text { for all } g \in G, \quad A \in \mathscr{B} .
$$

Such a pair $(U, E)$ is called an imprimitivity system.

Consider a strongly continuous, separable and measurable $G$-valued stochastic process $\{\omega(t), t \in \mathbb{R}\}$ defined on a probability space $(\Omega, F, \mu)$ and satisfying the following conditions : (1) $\omega(0)=e$ with probability one; (2) $\omega(t)$ and $\omega(t)^{-1}$ have the same distribution for all $t$; (3) for disjoint intervals $\left.] t_{j}, s_{j}\right]$ the random variables $\omega\left(s_{j}\right) \omega\left(t_{j}\right)^{-1}$ are independent; (4) the distribution of $\omega(r+s) \omega(r+t)^{-1}$ does not depend on $r$ whenever $s \geqq t$ are fixed. Such a process is called a symmetric $G$-valued process with stationary independent increments. We denote by $F_{s, t}$ the smallest $\sigma$-algebra with respect to which all the random variables $\omega(a) \omega(b)^{-1}, t \leqq a \leqq b \leqq s$ are measurable.

Regarding the existence of a large class of such processes with independent increments the reader may refer to $[9,11]$.

Let $h=L_{2}\left(\mu, h_{0}\right)$ be the Hilbert space of all $h_{h_{0}}$-valued $\mu$-square integrable maps. We denote by $N$ and $N_{s, t}$ respectively the von Neumann algebras of all bounded operators which are multiplications by $F$ - and $F_{s, t}$-measurable $h_{0}$-operator valued functions. For any $\omega \in \Omega$ and $t \in \mathbb{R}$ define $\tilde{\omega}$ and $\omega^{t} \in \Omega$ by putting $\tilde{\omega}(s)=\omega(s)^{-1}$; $\omega^{t}(s)=\omega(s+t) \omega(t)^{-1}$ for all $s \in \mathbb{R}$. Define the automorphisms $\gamma_{t}, t \in \mathbb{R}$ and $\varrho$ of $N$ by putting

$$
\left(\gamma_{t}(A) f\right)(\omega)=A\left(\omega^{t}\right) f(\omega) ; \quad(\varrho(A) f)(\omega)=A(\tilde{\omega}) f(\omega)
$$

for every $A \in N$. Define the map $j: N \rightarrow B\left(h_{0}\right)$ by the Bochner integral

$$
j(A) \xi=\int A(\omega) \xi d \mu(\omega), \quad \xi \in h_{0} .
$$

Let $\left\{U_{s, t}, s \geqq t\right\}$ be the operators on $h$ defined by

$$
\left(U_{s, t} f\right)(\omega)=U_{\omega(s) \omega(t)^{-1}} f(\omega), \quad f \in h .
$$

Then $\left(N,\left(N_{s, t}\right), \gamma_{t}, \varrho\right)$ is a reflective covariant system, $j$ is a reflective covariant reducing map and $\left(U_{s, t}\right)$ is a reflectively covariantly adapted evolution.

Suppose $V$ is a bounded Borel function on $(X, \mathscr{B})$ and $B$ is the operator of multiplication by the constant $h_{0}$-operator valued function $\int V(x) E(d x)$. Then an easy computation using (4.1) shows that

$$
\left(U_{s, t} B U_{s, t}^{-1} f\right)(\omega)=\int V\left(\omega(t) \omega(s)^{-1} x\right) E(d x) f(\omega) .
$$

It follows from Theorem 2.1 that the cocycle determined by $B$ is given by

$$
\left(M_{s, t}^{B} f\right)(\omega)=\left[\int \exp \left\{-\int_{t}^{s} V\left(\omega(\tau) \omega(s)^{-1} x\right) d \tau\right\} E(d x)\right] f(\omega) .
$$


Since $j(B)=\int V(x) E(d x)$, Theorem $3.2(\mathrm{c})$ implies that

$$
\begin{gathered}
e^{-(s-t) H_{0}} \xi=\int\left[U_{\omega(s) \omega(t)^{-1}} \xi\right] d \mu(\omega), \\
e^{-(s-t)\left(H_{0}+\int V(x) E(d x)\right)} \xi \\
\left.=\int\left\{\iint \exp \left\{-\int_{t}^{s} V\left(\omega(\tau) \omega(s)^{-1} x\right) d \tau\right\} E(d x)\right] U_{\omega(s) \omega(t)-1} \xi\right\} d \mu(\omega)
\end{gathered}
$$

for all $\xi \in h_{0}$ where integration with respect to $\mu$ is in the Bochner sense. When $X=G=\mathbb{R}, \omega(t)$ is standard Brownian motion, $t \rightarrow U_{t}$ is the translation representation in $L_{2}(\mathbb{R})$ and $E(A)$ is multiplication by $\chi_{A}$, Eq. (4.2) and (4.3) describe the classical Feynman-Kac formula with $H_{0}=p^{2} / 2$ and $\int V(x) E(d x)=V(q)$.

\section{Time Orthogonal Unitary Dilations}

To begin with we consider the case of a strongly continuous self-adjoint contraction semigroup $\left(A_{t}, t \geqq 0\right)$ with a bounded positive self-adjoint infinitesimal generator $H$ in a Hilbert space $\hbar_{0}$. Let $h=L_{2}\left(\mathbb{R}, h_{0}\right)$ be the Hilbert space of all $h_{0}$-valued square integrable maps on $\mathbb{R}$ and let the shifts $\left\{S_{t}, t \in \mathbb{R}\right\}$ and reflection $S$ be defined on $\ell$ by

$$
\left(S_{t} f\right)(s)=f(s-t) ; \quad(S f)(s)=f(-s), \quad f \in h, \quad t \in \mathbb{R} .
$$

For any Borel set $A \subseteq \mathbb{R}$ we write $h_{A}$ for the subspace of $h$ comprising of functions with support in $A$. Consider the Hilbert space $h_{1}=h_{0} \oplus h$ and denote by $J$ the imbedding $\zeta \rightarrow\left(\begin{array}{l}\zeta \\ 0\end{array}\right)$ from $h_{0}$ into $h$. Define the operators $\left\{U_{s, t}, s \geqq t\right\}$ in $h_{1}$ by

$$
U_{s, t}\left(\begin{array}{l}
\zeta \\
f
\end{array}\right)=\left(\begin{array}{cc}
A_{s, t} & B_{s, t} \\
C_{s, t} & I+D_{s, t}
\end{array}\right)\left(\begin{array}{l}
\zeta \\
f
\end{array}\right)
$$

where $A_{s, t}, B_{s, t}, C_{s, t}$ and $D_{\mathrm{s}, t}$ are operators from $h_{0}$ into $h_{0}, h$ into $h_{0}, h_{0}$ into $h$ and $h$ into $h_{0}$ respectively determined by the equations

$$
\left.\begin{array}{l}
A_{s, t}=A_{s-t} ; \quad B_{s, t} f=-(2 H)^{1 / 2} \int \chi_{[t, s]}(x) A_{s-x} f(x) d x ; \\
\left.C_{s, t}\right)(x)=\chi_{[t, s]}(x)(2 H)^{1 / 2} A_{x-t} \zeta ; \\
\left(D_{s, t} f\right)(x)=-2 \int \chi_{\mathrm{j}, s]}(x) \chi_{] y, s]}(x) H A_{x-y} f(y) d y .
\end{array}\right\}
$$

Lemma 5.1. For $r \geqq s \geqq t$,

$$
U_{r, s} U_{s, t}=U_{r, t} .
$$

Proof. It is immediate from the definitions (5.3) that $A_{r, s} A_{s, t}=A_{r, t}$ and $B_{r, s} C_{s, t}=B_{r, s} D_{s, t}=D_{r, s} C_{s, t}=0$. Since $H^{1 / 2}$ and $A_{s, t}$ commute for all $s \geqq t$ we have from a routine computation

$$
\begin{aligned}
& A_{r, s} B_{s, t}+B_{r, s}+B_{r, s} C_{s, t}=B_{r, t}, \\
& C_{r, s} A_{s, t}+C_{s, t}+D_{r, s} C_{s, t}=C_{r, t} .
\end{aligned}
$$


We shall now complete the proof by showing that

$$
C_{r, s} B_{s, t}+D_{r, s}+D_{s, t}+D_{r, s} D_{s, t}=D_{r, t} .
$$

To this end we observe that the last term on the left hand side of the above equation is 0 and the kernels of the first three operator terms are given by

$$
\begin{aligned}
& K_{1}(x, y)=-2 \chi_{] s, r]}(x) \chi_{] s, z]}(y) H A_{x-y}, \\
& K_{2}(x, y)=-2 \chi_{] s, r]}(y) \chi_{1 y, r]}(x) H A_{x-y}, \\
& K_{3}(x, y)=-2 \chi_{j t, s]}(y) \chi_{j y, s]}(x) H A_{x-y},
\end{aligned}
$$

respectively. A decomposition of the triangular region $\{(x, y): t<y \leqq x<r\}$ into a square and two triangular regions shows that

$$
\chi_{[t, r]}(y) \chi_{] y, r]}(x)=\chi_{] s, r]}(x) \chi_{j s, t]}(y)+\chi_{] s, r]}(y) \chi_{] y, r]}(x)+\chi_{] t, s]}(y) \chi_{] y, s]}(x),
$$

and hence

$$
\sum_{i=1}^{3} K_{i}(x, y)=-2 \chi_{] t, r]}(y) \chi_{] y, r]}(x) H A_{x-y},
$$

which is also the kernel of $D_{r, t^{-}}$This proves (5.4).

Lemma 5.2. For all $r \geqq s \geqq t$

$$
\begin{aligned}
& \left(\begin{array}{ll}
I & 0 \\
0 & S
\end{array}\right) U_{s, t}\left(\begin{array}{cc}
I & 0 \\
0 & S_{r}^{-1}
\end{array}\right) \\
& \left(\begin{array}{ll}
I & 0 \\
0 & S
\end{array}\right) U_{s, t}^{*}\left(\begin{array}{cc}
I & 0 \\
0 & S^{-1}
\end{array}\right)=U_{-t,-s, r+t}
\end{aligned}
$$

Proof. To prove (5.6) we first observe that $A_{s, t}=A_{s-t}$ and for any $f \in h$ we have by a change of variables

$$
\begin{aligned}
B_{s, t} S_{r}^{-1} f & =-(2 H)^{1 / 2} \int \chi_{[t, s]}(x) A_{s-x} f(x+r) d x \\
& =-(2 H)^{1 / 2} \int \chi_{j r+t, r+s]}(x) A_{r+s-x} f(x) d x \\
& =B_{r+s, r+t} f .
\end{aligned}
$$

Similarly we have

$$
S_{r} C_{s, t}=C_{r+s, r+t} .
$$

Further for any $x \in \mathbb{R}$ we have

$$
\begin{aligned}
{\left[S_{r} D_{s, t} S_{r}^{-1} f\right](x) } & =\left(D_{s, t} S_{r}^{-1} f\right)(x-r) \\
& =-2 \int \chi_{] t, s]}(y) \chi_{] y, s]}(x-r) H A_{x-r-y} f(y+r) d y \\
& =-2 \int \chi_{] t, s]}(y-r) \chi_{] y-r, s]}(x-r) H A_{x-y} f(y) d y \\
& =-2 \int \chi_{] r+i, r+s]}(y) \chi_{[y, s+r]}(x) H A_{x-y} f(y) d y \\
& =\left[D_{r+s, r+t} f\right](x) .
\end{aligned}
$$

Now (5.6) follows from (5.8) (5.10). 
To prove (5.7) we have to only observe that the adjoints of the operators $A_{s, t}$, $B_{s, t}, C_{s, t}$, and $D_{s, t}$ are given by

$$
\left.\begin{array}{rl}
A_{s, t}^{*} & =A_{s, t} ; \quad\left(\mathrm{B}_{s, t}^{*}, t\right)(x)=-\chi_{] t, s[}(x)(2 H)^{1 / 2} A_{s-x} \zeta ; \\
C_{s, t}^{*} f & =(2 H)^{1 / 2} \int \chi_{j t, s[}(x) A_{x-t} f(x) d x ; \\
& =-2 \int \chi_{1 t, s[}(y x) \chi_{[x, s]}(y) H A_{y-x} f(y) d y,
\end{array}\right\}
$$

respectively and follow a procedure similar to the proof of (5.6).

Lemma 5.3. $U_{\mathrm{s}, \mathrm{t}}$ is unitary.

Proof. Since $H^{1 / 2}$ and $A_{t}$ commute we have for $\zeta \in h_{0}$, using (5.11)

$$
\begin{aligned}
& \left(A_{s, t}^{*} A_{s, t}+C_{s, t}^{*} C_{s, t}\right) \zeta \\
& \quad=A_{2(s-t)} \zeta+2 H \int \chi_{j t, s[}(x) A_{2(x-t)} \zeta d x \\
& \quad=A_{2(s-t)} \zeta-\int \chi_{j t, s[}(x) \frac{d}{d x} A_{2(x-t)} \zeta d x=\zeta .
\end{aligned}
$$

Also, for any $f \in h$,

$$
\begin{aligned}
\left(A_{s, t}^{*} B_{s, t}+C_{s, t}^{*}+C_{s, t}^{*} D_{s, t}\right) f & \\
= & -A_{(s-t)}(2 H)^{1 / 2} \int \chi_{] t, s[}(x) A_{s-x} f(x) d x+(2 H)^{1 / 2} \int \chi_{] t, s[}(x) A_{x-t} f(x) d x \\
& -2(2 H)^{1 / 2} \int \chi_{[t, s]}(x) A_{x-t} \int \chi_{] t, s]}(y) \chi_{] y, s]}(x) H A_{x-y} f(y) d y d x \\
= & (2 H)^{1 / 2}\left\{\int \chi_{] t, s]}(x)\left[-A_{2 s-t-x}+A_{x-t}\right] f(x) d x\right. \\
& \left.-\int \chi_{] t, s]}(y)\left\{\int \chi_{] y, s]}(x) 2 H A_{2 x-t-y} f(y) d x\right\} d y\right\} .
\end{aligned}
$$

The right hand side of the last equation is identically zero in view of the identity $2 H A_{2 x-t-y}=-\frac{d}{d x} A_{2 x-t-y}$. Thus

$$
A_{s, t}^{*} B_{s, t}+C_{s, t}^{*}+C_{s, t}^{*} D_{s, t}=0 .
$$

Next we observe that $D_{s, t}^{*} D_{s, t}$ is an integral operator in $h=L^{2}\left(\mathbb{R}, h_{0}\right)$ with kernel

$$
\begin{aligned}
K(x, y) & =4 \int \chi_{] z, s]}(x) \chi_{[x, s]}(z) H A_{z-x} \chi_{] t, s]}(y) \chi_{] y, s]}(z) H A_{t-y} d z \\
& =2 \chi_{] t, s]}(x) \chi_{] t, s]}(y) H\left[A_{|x-y|}-A_{2 s-x-y}\right] .
\end{aligned}
$$

The kernel of the integral operator $B_{s, t}^{*} B_{s, t}$ is

$$
(2 H)^{1 / 2} \chi_{j t, s]}(x) A_{s-x}(2 H)^{1 / 2} \chi_{[t, s]}(y) A_{s-y}=2 \chi_{] t, s]}(x) \chi_{[t, s]}(x) \chi_{[t, s]}(y) H A_{2 s-x-y} .
$$

The kernel of $D_{s, t}^{*}+D_{s, t}$ is

$$
\begin{aligned}
& 2 \chi_{] l, s]}(x) \chi_{[x, s]}(y) H A_{y-x}+2 \chi_{[t, s]}(y) \chi_{1 y, s \mathrm{~s}}(x) A_{x-y} \\
& \quad=2 \chi_{[t, s]}(x) \chi_{[j, s]}(y) H A_{|x-y|} .
\end{aligned}
$$

From (5.14)-(5.16) we conclude that

$$
B_{s, t}^{*} B_{s, t}+D_{s, t}^{*}+D_{s, t}+D_{s, t}^{*} D_{s, t}=0
$$


Equations (5.12), (5.13), and (5.17) imply that $U_{s, t}^{*} U_{s, t}=I$. We use (5.7) to observe that

$$
\begin{aligned}
U_{s, t} U_{s, t}^{*} & =\left(\begin{array}{ll}
I & 0 \\
0 & S
\end{array}\right) U_{-t,-s}^{*}\left(\begin{array}{cc}
I & 0 \\
0 & S^{-1}
\end{array}\right)\left(\begin{array}{cc}
I & 0 \\
0 & S^{-1}
\end{array}\right) U_{-t,-s}\left(\begin{array}{ll}
I & 0 \\
0 & S
\end{array}\right) \\
& =\left(\begin{array}{ll}
I & 0 \\
0 & S
\end{array}\right) U_{-t,-s}^{*} U_{-t,-s}\left(\begin{array}{ll}
I & 0 \\
0 & S
\end{array}\right)=I . \square
\end{aligned}
$$

Lemma 5.4. In the decomposition $h_{1}=\left(h_{0} \oplus h_{1 t, s]}\right) \oplus h_{j t, s]}^{\perp}$ where $h_{i t, s]}^{\perp}$ is the orthogonal complement of $h_{\mathrm{j} t, s]}$ in $h$, the operator $U_{s, t}$ assumes the form

$$
U_{s, t}=V_{s, t} \oplus I,
$$

where $V_{s, t}$ is a unitary operator in $h_{0} \oplus h_{1, s]}$.

Proof. Let $E$ be the projector onto $h_{[s, t]}$ in $h$. Since

$$
B_{s, t} E=B_{s, t} ; \quad E C_{s, t}=C_{s, t} ; \quad E D_{s, t}=D_{s, t} E=D_{s, t},
$$

we can write

$$
U_{s, t}=\left(\begin{array}{cc}
A_{s, t} & B_{s, t} \\
C_{s, t} & I+D_{s, t}
\end{array}\right)=\left(\begin{array}{ll}
I & 0 \\
0 & E
\end{array}\right)\left(\begin{array}{cc}
A_{s, t} & B_{s, t} \\
C_{s, t} & I+D_{s, t}
\end{array}\right)\left(\begin{array}{ll}
I & 0 \\
0 & E
\end{array}\right)+\left(\begin{array}{cc}
0 & 0 \\
0 & I-E
\end{array}\right)
$$

and hence the lemma is proved.

Theorem 5.5. Let $H$ be a positive self-adjoint operator in a Hilbert space $\mathscr{h}_{0}$. Let $h=L_{2}\left(\mathbb{R}, h_{0}\right), h_{1}=h_{0} \oplus h$ and let $J: \zeta \rightarrow\left(\begin{array}{l}\zeta \\ 0\end{array}\right)$ be the natural imbedding of $h_{0}$ in $h_{1}$. Then there exists a strongly continuous unitary evolution $\left\{U_{s, t}, s \geqq t\right\}$ in hatisfying the following: (a) $J^{*} U_{s, t} J=e^{(t-s) H}$; (b) in the decomposition

$$
h_{1}=\left(h_{0} \oplus h_{j t, s]}\right) \oplus h_{\mathrm{it}, s]}^{\perp}
$$

where $h_{1, s]}$ is the subspace of $h$ consisting of functions with support $\left.] t, s\right]$ and $h_{j t, s]}^{1}$ is the orthogonal complement of $h_{1, s]}$ in $h, U_{s, t}=V_{s, t} \oplus I$ where $V_{s, t}$ is a unitary operator in $h_{0} \oplus h_{l t, s]}$; (c) $\left(I \oplus S_{r}\right) U_{s, t}\left(I \oplus S_{r}\right)^{-1}=U_{r+s, r+t}$ where $I \oplus S_{r}$ is the direct sum of the identity in $\hbar_{0}$ and $S_{r}$ is the shift defined by (5.1) in $h$; (d) $(I \oplus S) U_{s, t}^{*}(I \oplus S)^{-1}=U_{-t,-s}$ where $I \oplus S$ is the direct sum of the identity in $\hbar_{0}$ and $S$, the reflection defined by (5.1) in $\hbar$.

Proof. First of all we observe that we may assume without loss of generality that the operator $H$ is bounded. For if not, we may write $h_{0}$ as a direct $\operatorname{sum} \bigoplus_{n=1}^{\infty} h^{(n)}$ such that $H$ is bounded in each $\ell^{(n)}$, construct the evolutions $U_{s, t}^{(n)}$ in $\ell_{0}^{(n)} \oplus L_{2}\left(\mathbb{R}, \ell_{0}^{(n)}\right)$ and put the pieces together without any difficulty. When $H$ is bounded we construct $U_{s, t}$ by putting $A_{s, t}=e^{(t-s) k}$ and using (5.2) and (5.3). Then we conclude from Lemmas 5.1-5.4 that $U_{s, t}$ is a unitary evolution satisfying properties (1)-(4) of the theorem. Thus only strong continuity remains to be established. Because of unitarity this is equivalent to establishing the weak continuity of $U_{s, t}$ and hence 
the weak continuity of the operators $A_{s, t}, B_{s, t}, C_{s, t}$, and $D_{s, t}$ defined by (5.3). By assumption $A_{s, t}$ is strongly, hence weakly continuous. If $\zeta \in h_{0}, f, g \in h_{t}$ we have

$$
\begin{aligned}
\left\langle\zeta, B_{s, t} f\right\rangle & =-2^{1 / 2} \int \chi_{] t, s]}(x)\left\langle\zeta, H^{1 / 2} A_{s-x} f(x)\right\rangle d x \\
& =-2^{1 / 2} \int_{t}^{s}\left\langle\zeta, H^{1 / 2} A_{s-x} f(x)\right\rangle d x .
\end{aligned}
$$

Since the right hand side is continuous in the variables $t$ and $s$, the weak continuity of $B_{s, t}$ follows. A similar argument holds for the remaining operators.

In view of the properties (1) (4) of the evolution $\left\{U_{s, t}, s \geqq t\right\}$ we call it a timeorthogonal unitary dilation of the semigroup $\left\{e^{-t H}, t \geqq 0\right\}$, and we note that it is reflective and covariant under time inversion and translations. It may be remarked that the dilation of Theorem 5.5 is different from the classical dilations of one parameter contraction semigroups of operators in a Hilbert space into one parameter groups of unitary operators in a larger Hilbert space (see $[14,4,10]$ ). The replacement of invariance under time translation by covariance and the time orthogonality property in Theorem 5.5 (b) are the essential features of the new construction.

\section{Second Quantisation}

Let $h$ be a Hilbert space. The (symmetric) Fock space [6] over $h$ is the direct sum

$$
\Gamma(h)=\bigoplus_{n=0}^{\infty} h^{(n)},
$$

where $h^{(0)}=\mathbb{C}$ and for $n \geqq 1$ the $n$-particle space $h^{(n)}$ is the symmetric component of the $n$-fold tensor product of $h$ with itself. The exponential vectors, [6]

$$
e(f)=\left(1, f,(2 !)^{-1 / 2} f \otimes f,(3 !)^{-1 / 2} f \otimes f \otimes f, \ldots\right), \quad f \in h
$$

form a total set in $\Gamma(h)$; the vacuum, denoted by $\Omega$ or $\Omega(h)$, is the vector

$$
e(0)=(1,0,0, \ldots) \text {. }
$$

If $h=h_{1} \oplus \ldots \oplus h_{n}$ is a direct sum then there is a Hilbert space isomorphism, which we use to identify the two spaces, from $\Gamma\left(h_{h}\right)$ to $\Gamma\left(h_{1}\right) \otimes \ldots \otimes \Gamma\left(h_{n}\right)$ under which each exponential vector $e\left(f_{1}, \ldots, f_{n}\right)$ is mapped to the product vector $e\left(f_{1}\right) \otimes \ldots \otimes e\left(f_{n}\right)$.

Let $C$ be a contraction from $h$ to a second Hilbert space $h$. The second quantisation $\Gamma(C)$ [5] is a contraction from $\Gamma(h)$ to $\Gamma(h)$ whose action on exponential vectors is

$$
\Gamma(C) e(f)=e(C f) .
$$

The map $C \mapsto \Gamma(C)$ is continuous for the strong operator topologies in $\mathbf{B}\left(h, h^{\prime}\right)$ and $\mathbf{B}\left(\Gamma(h), \Gamma\left(h^{\prime}\right)\right)$. Second quantisation satisfies the functorial rules

$$
\begin{aligned}
\Gamma(I) & =I, \\
\Gamma\left(C_{1} C_{2}\right) & =\Gamma\left(C_{1}\right) \Gamma\left(C_{2}\right), \\
\Gamma\left(C^{*}\right) & =\Gamma(C)^{*} .
\end{aligned}
$$


From (6.1) it is clear that, if $C=C_{1} \oplus \ldots \oplus C_{n}$ is a direct sum, then

$$
\Gamma(C)=\Gamma\left(C_{1}\right) \otimes \ldots \otimes \Gamma\left(C_{n}\right) .
$$

Let $J$ be the inclusion injection from $\ell$ into a larger space $\ell^{\prime}$. Then, for $A \in \mathbf{B}\left(\Gamma\left(\ell^{\prime}\right)\right)$, $\Gamma(J)^{*} A \Gamma(J)$ is the vacuum conditional expectation of $A$ given $\mathbf{B}(\Gamma(\ell))$, that is, the unique operator in $\mathbf{B}(\Gamma(h))$ such that, for arbitrary $\psi, \chi \in \Gamma(h)$,

$$
\left\langle\psi, \Gamma(J)^{*} A \Gamma(J) \chi\right\rangle=\left\langle\psi \otimes \Omega\left(h^{\perp}\right), A \chi \otimes \Omega\left(h^{\perp}\right)\right\rangle,
$$

where $h^{\perp}$ is the orthogonal complement of $h$ in $h^{\prime}$ and we have used the identifications

$$
h^{\prime}=h h^{\perp \perp}, \quad \Gamma\left(h^{\prime}\right)=\Gamma(h) \otimes \Gamma\left(h^{\perp}\right) .
$$

The Fock representation of the canonical commutation relations (RCCR) over $h$ is the family of unitary Weyl operators $(W(f): f \in h)$ in $\Gamma(h)$, whose actions on exponential vectors are

$$
W(f) e(g)=\exp \left(-\frac{1}{4}\|f\|^{2}+2^{-1 / 2} i\langle f, g\rangle\right) e\left(g+2^{-1 / 2} i f\right) .
$$

The map $f \mapsto W(f)$ is continuous in the norm topology of $h$ and the strong operator topology of $\mathbf{B}(\Gamma(h))$, the Weyl relation

$$
W(f) W(g)=\exp \left\{-\frac{1}{2} i \operatorname{Im}\langle f, g\rangle\right\} W(f+g) \quad(f, g \in h)
$$

holds and the vacuum expectation functional is

$$
F(f)=\langle\Omega, W(f) \Omega\rangle=\exp \left(-\frac{1}{4}\|f\|^{2}\right) .
$$

The Fock representation is irreducible [6]. If $U$ is a unitary operator in $h$ then, for $f \in h$,

$$
\Gamma(U) W(f) \Gamma(U)^{-1}=W(U f) .
$$

If $h$ is a direct sum, $h=h_{1} \oplus \ldots \oplus h_{n}$, then, for $f=\left(f_{1}, \ldots, f_{n}\right) \in h$

$$
W(f)=W\left(f_{1}\right) \otimes \ldots \otimes W\left(f_{n}\right) .
$$

Now let $h_{0}$ be a Hilbert space, let $h=L^{2}\left(\mathbb{R}, h_{0}\right)$ be the Hilbert space of $h_{0}$-valued square-integrable measurable vector-valued functions and let $h_{1}=h_{0} \oplus h$. Let $N$ be the von Neumann algebra $\mathbf{B}\left(\Gamma\left(h_{1}\right)\right)$ and, for $s \geqq t$, let $N_{s, t}$ be the von Neumann algebra generated by the operators $W(\zeta, f), \zeta \in h_{0}, f \in h_{\mathrm{j}, t]}$, where $h_{3 s, t]}$ is the subspace of $h$ comprising functions vanishing outside $\left.] t, s\right]$.

Next let $S_{t}, t \geqq 0$ and $S$ be the shift operators and reflection in $h$ and define automorphisms $\gamma_{t}, t \geqq 0$, and $\varrho$ of $N$ by

$$
\begin{aligned}
\gamma_{t}(A) & =\Gamma\left(I \oplus S_{t}\right) A \Gamma\left(I \oplus S_{t}\right)^{-1} \\
\varrho(A) & =\Gamma(I \oplus S) A \Gamma(I \oplus S)^{-1} .
\end{aligned}
$$

Then using (6.5), (6.7) and the fact that $\mathbf{B}\left(\Gamma\left(h_{1}\right)\right)$ is generated by the irreducible family of Weyl operators, it can be seen that $\left(N,\left(N_{s, t}\right),\left(\gamma_{t}\right), \varrho\right)$ is a reflective covariant system.

Next let $J$ be the embedding $\zeta \rightarrow(\zeta, 0)$ of $h_{0}$ in $h_{1}$ and define $j: N \rightarrow \mathbf{B}\left(\Gamma\left(h_{0}\right)\right)$ by

$$
j(A)=\Gamma\left(J^{*}\right) A \Gamma(J),
$$


thus $j(A)$ is the conditional expectation of $A$ given $\mathbf{B}\left(\Gamma\left(h_{0}\right)\right)$. Finally let $\left(U_{\mathrm{s}, t}\right)$ be the time orthogonal unitary dilation of a strongly continuous self-adjoint contraction semigroup acting in $h_{0}$ as in Theorem 5.5.

Theorem 6.1. $j$ is a reflective covariant reducing map and $\left(\Gamma\left(U_{s, t}\right), s \geqq t\right)$ is a reflectively covariantly adapted evolution for $\left(N,\left(N_{s, t}\right),\left(\gamma_{t}\right), \varrho\right)$.

Proof. That $j\left(A^{*}\right)=j(A)^{*}$ is clear from (6.4). To prove that $j$ satisfies (3.1) we make the natural identifications

$$
\begin{aligned}
h_{h} & =h_{] a, b]} \oplus h_{] c, d]} \oplus\left(h_{] a, b]} \oplus h_{\mathrm{Jc}, d]}\right)^{\perp} \\
h_{1} & =h_{0} \oplus h_{] a, b]} \oplus h_{] c, d]} \oplus\left(h_{] a, b]} \oplus h_{] c, d]}\right)^{\perp} \\
N_{a, b} & =\left\{W(\zeta) \otimes W\left(f_{] a, b]}\right) \otimes I \otimes I: \zeta \in h_{0}, f \in h_{] a, b]}\right\}^{\prime \prime} \\
& =\mathbf{B}\left(\Gamma\left(h_{0}\right) \otimes \Gamma\left(h_{1 a, b]}\right)\right) I \otimes I \\
& =\mathbf{B}\left(\Gamma\left(h_{0}\right)\right) \otimes \mathbf{B}\left(\Gamma\left(h_{1 a, b]}\right) \otimes I \otimes I,\right.
\end{aligned}
$$

and similarly

$$
\left.N_{c, d}=\mathbf{B}\left(\Gamma\left(h_{0}\right)\right) \otimes I \otimes \mathbf{B}\left(\Gamma\left(\ell_{c}\right], d\right]\right) \otimes I \text {. }
$$

Since the von Neumann algebras $\mathbf{B}\left(\Gamma\left(h_{] a, b]}\right)\right) \otimes I \otimes I$ and $I \otimes \mathbf{B}\left(\Gamma\left(h_{] c, d}\right)\right) \otimes I$ are independent in the vacuum state

$$
\left.\Omega(h)=\Omega\left(h_{] a, b]}\right) \otimes \Omega\left(h_{] c, d]}\right) \otimes \Omega\left(h_{] a, b]} \otimes h_{\mathrm{jc}, d]}\right)^{\perp}\right),
$$

(3.1) now follows from Theorem 1.1. Thus $j$ is a reducing map. By second quantising the relations

$$
\left(I \oplus S_{t}\right)^{-1} J=(I \oplus S)^{-1} J=J
$$

and using (6.3), we have

$$
\Gamma\left(I \oplus S_{t}\right)^{-1} \Gamma(J)=\Gamma(I \oplus S)^{-1} \Gamma(J)=\Gamma(J),
$$

and hence, taking adjoints,

$$
\Gamma(J)^{*} \Gamma\left(I \oplus S_{t}\right)=\Gamma(J)^{*} \Gamma(I \oplus S)=\Gamma(J)^{*} .
$$

From (6.8) and (6.9) it is clear that $j \circ \gamma_{r}=j \circ \varrho=j$ so that $j$ is reflective covariant.

Finally, it is clear from (6.2), (6.3) and the continuity of second quantisation that $\left(\Gamma\left(U_{s, t}\right), s \geqq t\right)$ is an evolution; second quantising (5.2) using (6.5) gives

$$
\Gamma\left(U_{s, t}\right)=I\left(V_{s, t}\right) \otimes I \in \mathbf{B}\left(\Gamma\left(h_{0} \oplus h_{1 t, s]}\right) \otimes I=N_{s, t},\right.
$$

so the evolution is adapted. That the adaptation is reflective and covariant follows from the second quantisations of (5.3) and (5.4).

The reduced evolution corresponding to the reducing map $j$ of the evolution $\left(\Gamma\left(U_{s, t}\right)\right)$ is easily found by second quantising the relation $A_{s-t}=J^{*} U_{s, t} J$ to be the second quantisation $\left(\Gamma\left(A_{t}\right): t \geqq 0\right)$ of the original semigroup $\left(A_{t}\right)$. We write

$$
\Gamma\left(A_{t}\right)=e^{-t H}
$$

Let us now consider perturbations. 
It is now clear that elements $B$ of $\bigcap_{s>t} N_{s, t}$ are precisely those of form $V \otimes I$ with $V \in \mathbf{B}\left(h_{0}\right)$, and such elements are automatically invariant under the $\gamma_{t}$. From Theorem 3.2(c) we may thus write, for self-adjoint $V \in \mathbf{B}\left(h_{0}\right)$,

$$
\begin{aligned}
e^{-(s-t)(H+V)} & =j\left(M_{s, t}^{V} \Gamma\left(U_{s, t}\right)\right) \\
& =\mathbb{E}\left[M_{s, t}^{V} \Gamma\left(U_{s, t}\right) \mathbf{B}\left(\Gamma\left(h_{0}\right)\right)\right],
\end{aligned}
$$

where $\left(M_{s, t}^{V}\right)$ is the cocycle for $\Gamma\left(U_{s, t}\right)$ determined by $B=V \otimes I$.

We investigate conditions under which the family of operators

$$
\Gamma\left(U_{s, t}\right) V \otimes I \Gamma\left(U_{s, t}\right)^{-1}
$$

is commutative, so that, according to Theorem 2.1, the cocycle can be expressed in integral form

$$
M_{s, t}^{V}=\exp \left\{-\int_{t}^{s} \Gamma\left(U_{s, \tau}\right) V \otimes I \Gamma\left(U_{s, \tau}\right)^{-1} d \tau\right\} .
$$

Let $K_{0}$ be a conjugation in $h_{0}$, that is a conjugate linear isometric map from $h_{0}$ to itself whose square is the identity, such that

$$
K_{0} A_{t}=A_{t} K_{0} \quad(t \geqq 0) .
$$

Then if $K$ is the conjugation in $h=L^{2}\left(\mathbb{R}, h_{0}\right)$ given by

$$
(K f)(x)=K_{0}(f(x))
$$

and $K_{1}=K_{0} \oplus K$, we have

$$
K_{1} J=J K_{0}
$$

and, for arbitrary $s \geqq t$,

$$
K_{1} U_{s, t}=U_{s, t} K_{1}
$$

Let $N_{0}, N_{1}$ be the von Neumann algebras generated by the operators

$$
\left\{W(\zeta): \zeta \in h_{0}, K_{0} \zeta=\zeta\right\}, \quad\left\{W(f): f \in h_{1}, K_{1} f=f\right\}
$$

respectively. Then, using (6.6), it is easily seen that $N_{0}$ and $N_{1}$ are commutative. Also, since by (6.7),

$$
\begin{aligned}
\Gamma\left(U_{s, t}\right) W(\zeta) \otimes I \Gamma\left(U_{s, t}\right)^{-1} & =\Gamma\left(U_{s, t}\right) W(J \zeta) \Gamma\left(U_{s, t}\right)^{-1} \\
& =\Gamma\left(U_{s, t} J \zeta\right),
\end{aligned}
$$

and if $K_{0} \zeta=\zeta$, then

$$
K_{1} U_{s, t} J \zeta=U_{s, t} K_{1} J \zeta=U_{s, t} J K_{0} \zeta=U_{s, r} J_{\zeta}^{\zeta},
$$

we see that conjugation by $\Gamma\left(U_{s, t}\right)$ maps $N_{0} \otimes I$ into the commutative algebra $N_{1}$. It follows that, for $V$ belonging to $N_{0}$, the operators $\Gamma\left(U_{s, t}\right) V \otimes I \Gamma\left(U_{s, t}\right)^{-1}$ commute so that by Theorem 2.1 the cocycle can be written in integral form so that $(6.10)$ becomes

$$
\left.e^{-(s-t)(H+V)}=\mathbb{E}\left[\exp \left\{-\int_{t}^{s} \Gamma\left(U_{s, \tau}\right) V \otimes I \Gamma\left(U_{s, \tau}\right)^{-1} d \tau\right\} \Gamma\left(U_{s, t}\right) \mid \mathbf{B}(\Gamma(h))\right)\right] .
$$


A conjugation $K_{0}$ in $h_{0}$ effects a separation of the set of fields $r(\zeta), \zeta \in h_{0}$, that is the self-adjoint operators defined by

$$
W(t \zeta)=e^{i t r(\zeta)}, \quad \zeta \in h_{0}, \quad t \in \mathbb{R}
$$

into "coordinate" fields, for which $K_{0} \zeta=\zeta$ and "momentum" fields, for which $K_{0} \zeta=-\zeta$. Thus we may say that the integral form (6.12) of Feynman-Kac formula is valid provided the perturbation $V$ is a function of the coordinate fields alone, in the sense of being an element of the von Neumann algebra generated by the spectral projectors of these fields alone.

\section{An Example}

We consider the case [8] of the semigroup $A_{t}=e^{-t}$ acting in the Hilbert space $h_{0}=\mathbb{C}$, with complex conjugation as the conjugation $K_{0}$. The second quantisation $\left(\Gamma\left(A_{t}\right)\right)$ is then the semigroup in $\Gamma(\mathbb{C})=l^{2}$ whose infinitesimal generator is the number operator

$$
a^{\dagger} a\left(z_{0}, z_{1}, z_{2}, \ldots\right)=\left(0, z_{1}, 2 z_{2}, \ldots\right)
$$

or equivalently the renormalised harmonic oscillator Hamiltonian $\frac{1}{2}\left(p^{2}+q^{2}-1\right)$, where $p$ and $q$ are the canonical pair defined by

$$
e^{i t p}=W(i t), \quad e^{i t q}=W(t), \quad t \in \mathbb{R}
$$

and

$$
a=2^{-1 / 2}(q-i p), \quad a^{\dagger}=2^{-1 / 2}(q+i p)
$$

We shall show that the operator $\Gamma\left(U_{s, t}\right)$ can be expressed formally as a product integral

$$
\Gamma\left(U_{s, t}\right)=\prod_{t}^{s} e^{i \sqrt{2}(p \otimes d Q-q \otimes d P)}
$$

where $(P, Q)$ is essentially the (Fock) canonical Wiener process of [3] of minimal variance $\sigma^{2}=1$. We recall that for $t \geqq 0$ the operators $P(t), Q(t)$ of the process are defined in $\Gamma\left(L^{2}(\mathbb{R})\right)$ by

$$
e^{i x P(t)}=W\left(i x \chi_{[0, t]}\right), \quad e^{i \pm Q(t)}=W\left(x \chi_{[0, t]}\right) \quad(x \in \mathbb{R}) ;
$$

for $t<0$ we define $P(t), Q(t)$ by

$$
e^{i x P(t)}=W\left(-i x \chi_{] t, 0]}\right), \quad e^{i x Q(t)}=W\left(-x \chi_{] t, 0]}\right),
$$

so that, for arbitrary $s>t$,

$$
P(s)-P(t)=(s-t)^{1 / 2} p_{s, t}, \quad Q(s)-Q(t)=(s-t)^{1 / 2} q_{s, t},
$$

where $\left(p_{s, t}, q_{s, t}\right)$ is the canonical pair defined by

$$
e^{i x p_{s, t}}=W\left(i x(s-t)^{-1 / 2} \chi_{] t, s]}\right), \quad e^{i x q_{s, t}}=W\left(x(s-t)^{-1 / 2} \chi_{] t, s]}\right) \quad(x \in \mathbb{R}) .
$$


To give meaning to the product integral (7.1) we first consider the formal "Riemann product" for a partition $A=\left\{t=t_{0}<t_{1}<\ldots<t_{n}=s\right\}$,

$$
\begin{aligned}
T_{A} & =\prod_{j=1}^{n} \exp \left\{i \sqrt{2}\left(p \otimes\left(Q\left(t_{j}\right)-Q\left(t_{j-1}\right)\right)-q \otimes\left(P\left(t_{j}\right)-P\left(t_{j-1}\right)\right)\right\}\right. \\
& =\prod_{j=1}^{n} \exp \left\{i \theta_{j}\left(p \otimes q_{j}-q \otimes p_{j}\right)\right\}
\end{aligned}
$$

where

$$
\theta_{j}=\left(2\left(t_{j}-t_{j-1}\right)\right)^{1 / 2}
$$

and $\left(p_{j}, q_{j}\right)$ is the canonical pair

$$
p_{j}=p_{t_{j-1}, t_{j}} ; \quad q_{j}=q_{t_{j-1}, t_{j}} .
$$

Recognising $p \otimes q_{j}-q \otimes p_{j}$ as an angular momentum, we may define $\exp \left\{i \theta_{j}\left(p \otimes q_{j}-q \otimes p_{j}\right)\right\}$ rigorously as the second quantisation $\Gamma\left(R_{j}\right)$ of the rotation $R_{j}$ which acts in

$$
\mathbb{C} \oplus L^{2}(\mathbb{R})=\mathbb{C} \oplus \mathbb{C} \chi_{j} \oplus\left(\mathbb{C} \chi_{j}\right)^{\perp},
$$

where

$$
\chi_{j}=\left(t_{j}-t_{j-1}\right)^{-1 / 2} \chi_{]_{j-1}, t_{j}\right]},
$$

as

$$
R_{j}(z, f)=\left(z_{0}, f_{0}\right)
$$

where

$$
\begin{aligned}
& z_{0}=\cos \theta_{j} z-\sin \theta_{j}\left\langle\chi_{j}, f\right\rangle \\
& f_{0}=\sin \theta_{j} z \chi_{j}+\left(\cos \theta_{j}-1\right)\left\langle\chi_{j}, f\right\rangle \chi_{j}+f .
\end{aligned}
$$

Hence

$$
\prod_{j=1}^{n} R_{j}(z, f)=\left(z_{1}, f_{1}\right)
$$

where

$$
\begin{aligned}
z_{1}= & \left(\prod_{j=1}^{n} \cos \theta_{j}\right) z-\sum_{j=1}^{n}\left\langle\chi_{j} f\right\rangle \sin \theta_{j} \prod_{k=j+1}^{n}\left(\cos \theta_{k}\right) \\
f_{1}= & \sum_{j=1}^{n} \sin \theta_{j}\left(\prod_{k=1}^{j-1} f_{k} \sin \theta_{k}-\sum_{k=1}^{j-1} f_{k} \sin \theta_{k} \prod_{l=k+1}^{j-1}\left(\cos \theta_{l}\right)\right) \chi_{j} \\
& +\sum_{j=1}^{n}\left(\cos \theta_{j}-1\right) f_{j} \chi_{j}+f .
\end{aligned}
$$

From this it can be seen that, for sufficiently smooth $f$,

$$
\lim _{\Lambda} \prod_{j=1}^{n} R_{j}(z, f)=\left(z^{\prime}, f^{\prime}\right)
$$


where

$$
\begin{aligned}
z^{\prime} & =e^{-(s-t)} z-\sqrt{2} \int \chi_{[t, s]}(x) e^{-(s-x)} f(x) d x, \\
f^{\prime}(x) & =\sqrt{2} e^{-(x-t)} \chi_{[t, s]}(x) z-2 \int \chi_{[t, s]}(y) \chi_{[y, s]}(x) e^{-(x-y)} f(y) d y,
\end{aligned}
$$

and the limit is as the $\operatorname{mesh}|\boldsymbol{A}|=\max \left(t_{j}-t_{j-1}\right)$ decreases to zero. But this is precisely $U_{s, t}(z, f)$. Since $U_{s, t}$ is unitary the product $\prod_{j=1}^{n} R_{j}$ converges strongly to $U_{s, t}$. Using the strong continuity of second quantisation it follows that $\Gamma\left(U_{s, t}\right)$ is indeed given by the limit of the Riemann products $T_{A}=\prod_{j=1}^{n} \Gamma\left(R_{j}\right)$.

The algebra $N_{0}$ in this case is clearly the von Neumann algebra generated by the operators $e^{i x q}, x \in \mathbb{R}$, thus a self-adjoint element of $N_{0}$ takes the form $V(q)$ for some real, bounded measurable function $V$. Since, by (6.7)

where

$$
\begin{aligned}
\Gamma\left(U_{s, t}\right) e^{i x q} \otimes I \Gamma\left(U_{s, t}\right)^{-1} & =\Gamma\left(U_{s, t}\right) W(x, 0) \Gamma\left(U_{s, t}\right)^{-1} \\
& =W\left(U_{s, t}(x, 0)\right) \\
& =W\left(e^{-(s-t)} x, x f\right),
\end{aligned}
$$

$$
f(\tau)=\sqrt{2} \chi_{], s]}(\tau) e^{-(\tau-t)},
$$

we have

$$
\Gamma\left(U_{s, t}\right) V(q) \otimes I \Gamma\left(U_{s, t}\right)^{-1}=V\left(q_{t}^{s}\right),
$$

where $q_{t}^{s}$ is defined formally as

$$
q_{\mathrm{f}}^{s}=e^{-(s-t)} q \otimes I+\sqrt{2} I \otimes \int_{t}^{s} e^{-(\tau-t)} d Q(\tau),
$$

using the stochastic integral notation [3] for field operators associated with the canonical Wiener process; $q_{t}^{s}$ is rigorously defined by

$$
e^{i x q_{t}^{s}}=W\left(e^{-(s-t)} x, x f\right),
$$

with $f$ given by (7.2). Thus, from (6.12) we obtain the formula [7]

$e^{-(s-t)\left(\frac{1}{2}\left(p^{2}+q^{2}-1\right)+V(q)\right)}$

$$
=\mathbb{E}\left[\exp \left\{-\int_{t}^{s} V\left(e^{-(s-\tau)}\right) q \otimes I+\sqrt{2} I \otimes \int_{\tau}^{s} e^{-\left(\tau^{\prime}-\tau\right)} d Q\left(\tau^{\prime}\right)\right\} \prod_{t}^{s} e^{i \sqrt{2}(p \otimes d Q-q \otimes d P)} \mid \mathbf{B}\left(l_{2}\right)\right] .
$$

As was brought to our attention by a comment of L. van Hemmen, the Feynman-Kac formula (7.5) is essentially the "oscillator process" formula of [13, p. 52]. To see this, note first that, because of their relationship (7.4) to the $q$-component of the canonical Wiener process, in the state $\Omega_{0} \otimes \Omega\left(L^{2}(\mathbb{R})\right)$ where $\Omega_{0}$ is the oscillator ground state, the vacuum in $\Gamma(\mathbb{C})$, the commuting family of operators $q(t)=q_{t, 0}, t \geqq 0$, constitute a realisation of the Ornstein-Uhlenbeck velocity process. 
Moreover, for vectors in $l^{2}=\Gamma(\mathbb{C})$ expressed in the form $f(q) \Omega_{0}, g(q) \Omega_{0}$ we have, from (7.4) with $] t, s]$ replaced by $] 0, t]$,

$$
\begin{aligned}
\langle f & (q) \Omega_{0}, \exp \left\{-t\left(\frac{1}{2}\left(p^{2}+q^{2}-1\right)+V(q)\right\} g(q) \Omega_{0}\right\rangle \\
& =\left\langle\left(f(q) \Omega_{0}\right) \otimes \Omega, \exp \left\{-\int_{0}^{t} V(q(\tau)) d \tau\right\} \prod_{0}^{t} e^{i \sqrt{2}(p \otimes d Q-q \otimes d P)}\left(g(q) \Omega_{0}\right) \otimes \Omega\right\rangle \\
& =\left\langle f(q \otimes I) \Omega_{0} \otimes \Omega, \exp \left\{-\int_{0}^{t} V(q(\tau)) d \tau\right\} \prod_{0}^{t} e^{i \sqrt{2}(p \otimes d Q-q \otimes d P)} g(q \otimes I) \Omega_{0} \otimes \Omega\right\rangle \\
& =\left\langle f\left(q(0) \Omega_{0}\right) \otimes \Omega, \exp \left\{-\int_{0}^{t} V(q(\tau)) d \tau\right\} g(q(t)) \prod_{0}^{t} e^{i \sqrt{2}(p \otimes d Q-q \otimes d P)} \Omega_{0} \otimes \Omega\right\rangle,
\end{aligned}
$$

using (7.3),

$$
=\left\langle f(q(0)) \Omega_{0} \otimes \Omega, \exp \left\{-\int_{s}^{t} V(q(\tau)) d \tau\right\} g(q(t)) \Omega_{0} \otimes \Omega\right\rangle,
$$

using the invariance of the vacuum $\Omega_{0} \otimes \Omega$ under the second quantisation

$$
\begin{aligned}
\prod_{0}^{t} e^{i \sqrt{2}(p \otimes d Q-q \otimes d P)} & =\left\langle\Omega_{0} \otimes \Omega, \overline{f(q(0))} g(q(t)) \exp \left\{-\int_{0}^{t} V(q(\tau)) d \tau\right\} \Omega_{0} \otimes \Omega\right\rangle \\
& =\mathbb{E}\left[\overline{f(q(0))} g(q(t)) \exp \left\{-\int_{0}^{t} V(q(\tau)) d \tau\right\}\right],
\end{aligned}
$$

where the expectation is over the Ornstein-Uhlenbeck velocity process $(q(t))$. Thus, as in [13],

$$
\begin{aligned}
& \left\langle f(q) \Omega_{0}, \exp \left\{-t\left(\frac{1}{2}\left(p^{2}+q^{2}-1\right)+V(q)\right\} g(q) \Omega_{0}\right\rangle\right. \\
& \quad=\mathbb{E}\left[\overline{f(q(0))} g(q(t)) \exp \left\{-\int_{s}^{t} V(q(\tau)) d t\right\}\right] .
\end{aligned}
$$

From the viewpoint of noncommutative probability theory, it is natural to regard the operators

$$
q(t)=e^{-t} q \otimes I+\sqrt{2} I \otimes \int_{0}^{t} e^{-\tau} d Q(\tau), \quad t \geqq 0
$$

as the q-component of the canonical Ornstein-Uhlenbeck velocity process $(p(t), q(t), t \geqq 0)$, where

$$
p(t)=e^{-t} p \otimes I+\sqrt{2} I \otimes \int_{0}^{t} e^{-\tau} d P(\tau), \quad t \geqq 0 .
$$

Because $p(t)$ and $q(t)$ constitute a canonical pair for all $t \geqq 0$, the process $((p(t), q(t)))$ is essentially a noncommutative $W^{*}$-stochastic process in the sense of [2], insofar as the von Neumann uniqueness theorem gives rise to a family of von Neumann algebra isomorphisms $j(t), t \geqq 0$ from the von Neumann algebra generated by the spectral projectors of $p(t)$ and $q(t)$ to that generated by those of the fixed canonical pair $(p, q)$. 
The perturbation cocycle $\left(M_{t, 0}^{B}\right)$ corresponding to an element $B$ of $\mathbf{B}\left(l^{2}\right)$ not in $N_{0}$ can be expressed formally as the continuous product

$$
M_{t, 0}^{B}=\prod_{0}^{t} \exp \{-B(p(\tau), q(\tau)) d \tau\}
$$

here $B(p(\tau), q(\tau))$ is the pre-image of $B$ under $j(\tau)$.

\section{References}

1. Accardi, L.: On the quantum Feynman-Kac formula (preprint)

2. Accardi, L., Frigerio, A., Lewis, J.T.: Quantum stochastic processes (preprint)

3. Cockroft, A.M., Hudson, R.L.: Quantum mechanical Wiener processes. J. Multivar. Anal. 7, 107124 (1978)

4. Davies, E.B.: Some contraction semigroups in quantum probability. Z. Wahrsch. Verw. Geb. 23, 261-273 (1972)

5. Evans, D.E., Lewis, J.T.: Dilations of irreversible evolutions in algebraic quantum theory. Commun. Dublin Inst. for Adv. Studies, Series A 24 (1974)

6. Guichardet, A.: Symmetric Hilbert spaces and related topics. In: Lecture Notes in Mathematics, Vol. 261. Berlin, Heidelberg, New York: Springer 1972

7. Hudson, R.L., Ion, P.D.F.: The Feynman-Kac formula for a canonical quantum mechanical Wiener process, to appear in Proceedings of the Esztergom (1979) Colloquium "Random fields: rigorous results in statistical mechanics and quantum field theory"

8. Hudson, R.L., Ion, P.D.F., Parthasarathy, K.R.: The Feynman-Kac formula for Boson Wiener processes. In: Quantum mechanics in mathematics, physics, and chemistry. Gustafson, K., et al. (ed.). New York: Plenum Press 1981

9. Hunt, G.A. : Semigroups of measures on Lie groups. Trans. Am. Math. Soc. 81, 264-293 (1956)

10. Lax, P., Phillips, R.S.: Scattering theory. New York: Academic Press 1964

11. Parthasarathy, K.R.: Probability measures on metric spaces. New York: Academic Press 1967

12. Phillips, R.S.: Perturbation theory of semigroups of linear operators. Trans. Am. Math. Soc. 74, 199-220 (1953)

13. Simon, B.: Functional integration and quantum physics. New York: Academic Press 1979

14. Sz-Nagy, B., Foias, C.: Harmonic analysis of operators on Hibert space. Amsterdam: NorthHolland 1970

Communicated by H. Araki

Received April 15, 1981; in revised form July 26, 1981 